\title{
A Type Covering Lemma and the Excess Distortion Exponent for Coding Memoryless Laplacian Sources*
}

\author{
Yangfan Zhong, Fady Alajaji and L. Lorne Campbell \\ Department of Mathematics and Statistics \\ Queen's University, Kingston, ON K7L 3N6, Canada \\ Email: \{yangfan,fady,campbll1 $\} @$ mast.queensu.ca
}

\begin{abstract}
In this work, we introduce the notion of Laplaciantype class and derive a type covering lemma for the memoryless Laplacian source (MLS) under the magnitude-error distortion measure. We then present an application of the type covering lemma to the lossy coding of the MLS. We establish a simple analytical lower bound for the excess distortion exponent, namely, the exponent of the probability of representing the source beyond a given distortion threshold. It is noted that, by introducing the Laplacian-type class, one can employ the classical method of types to solve source coding and source-channel coding problems regarding the MLS.
\end{abstract}

\section{INTRODUCTION}

It is well known that the method of types is a very useful tool in information theory, particularly in Shannon theory, hypothesis testing and large deviation theory (e.g., [2], [3]). For a discrete memoryless source (DMS) with alphabet $\mathcal{S}$ and a given rational probability mass function $P$, the type$P$ class of $k$-length sequences $\mathbf{s} \triangleq\left(s_{1} s_{2} \cdots s_{k}\right) \in \mathcal{S}^{k}$ is the set of sequences that have single-symbol empirical distribution equal to $P$. Thus, by partitioning all sequences in $\mathcal{S}^{k}$ into type classes where the number of distinct classes grows subexponentially with $k$, the probability of a particular event (the probability of error, say) can be obtained by summing the probabilities of its intersections with the various type classes which decay exponentially as the sequence length $k$ approaches infinity [2]. However, such type model, in the sense of a common composition of single-symbol frequencies, cannot be implemented to sequences with continuous alphabets. When $\mathcal{S}$ is continuous, we need to find a counterpart to the type classes which partition the whole source space $\mathcal{S}^{k}$, while keeping an exponentially small probability in $k$.

In [1, Sec. VI. A], a continuous-alphabet analog to the method of types was studied for the memoryless Gaussian source (MGS) by introducing the notion of Gaussian-type classes. Given $\sigma^{2}>0$ and $\epsilon \in\left(0, \sigma^{2}\right)$, the Gaussian-type class, denoted by $\mathcal{T}^{\epsilon}\left(\sigma^{2}\right)$, is the set of all $k$-length sequences $\mathbf{s} \in \mathbb{R}^{k}$ such that

$$
\left|\mathbf{s}^{T} \mathbf{s}-k \sigma^{2}\right| \leq k \epsilon
$$

where $T$ is the transpose operation. Based on a sequence of $\left\{\sigma_{i}^{2}\right\}_{i=1}^{\infty}$, the Euclidean space $\mathbb{R}^{k}$ can be partitioned using (1), and it can be shown that for the zero-mean MGS, the probability of each type defined by (1) decays exponentially in $k$ [1]. As a result, it is possible to apply the method of

\footnotetext{
*This work was supported in part by PREA and NSERC of Canada.
}

types, adapted to the continuous-alphabet setting, to solve some source coding and joint source-channel coding problems regarding the MGS ([1], [9]).

In this work, we extend the concept of Gaussian-type classes [1]. Instead of using (1), we partition the whole Euclidean space $\mathbb{R}^{k}$ by a sequence of sets

$$
\mathcal{T}^{\epsilon}\left(\alpha_{i}\right) \triangleq\left\{\mathbf{s}:\left|\sum_{i=1}^{k}\right| s_{i}\left|-k \alpha_{i}\right| \leq k \epsilon\right\},
$$

associated with a sequence of positive numbers $\left\{\alpha_{i}\right\}_{i=1}^{\infty}$. We call each set $\mathcal{T}^{\epsilon}\left(\alpha_{i}\right)$ a Laplacian-type class with respect to $\alpha_{i}$, since the probability of each type class for a zero-mean memoryless Laplacian source (MLS) decays exponentially in $k$. Analogous to [1], we derive a type covering lemma (Lemma 3) under the magnitude-error distortion measure for Laplaciantype classes. Similarly, we can easily adapt the method of types to solve some source coding and joint source-channel coding problems (e.g., bounding the excess distortion exponent and source-channel excess distortion exponent with memoryless Gaussian noise channels) regarding the MLS. Due to the limited space, we only present an application of the type covering lemma to the lossy coding problem.

In image coding applications, the Laplacian distribution is often a good model to approximate the statistics of transform coefficients such as discrete cosine and wavelet transform coefficients ([7], [8]). Thus, it is of interest to study the theoretical performance for the lossy compression of Laplacian sources. In particular, we are interested in studying how fast the probability that the distortion due to source coding exceeds a certain tolerated threshold asymptotically decays to zero. Applying the type covering lemma for the Laplacian-type class, we establish a lower bound (see Theorem 1) for the exponent of excess distortion for the MLS. Note that the exponent of excess distortion for a general class of stationary memoryless sources was recently obtained in [4], where the exponent is expressed in Marton's form [6] (in terms of a minimized Kullback-Leibler divergence). We observe that our lower bound is indeed identical to this expression, and is therefore tight (see Corollary 1).

\section{PRELIMINARIES}

All logarithms and exponentials throughout this paper are in natural base. $\mathbb{E}(X)$ denotes the expectation of the random variable $(\mathrm{RV}) X . \Phi(\cdot)$ is the indicator function. 
We consider an MLS $P_{S}$ with alphabet $\mathcal{S}=\mathbb{R}$, mean zero, variance $2 \alpha^{2}$, and probability density function (pdf) $P_{S}(s)=$ $\frac{1}{2 \alpha} \exp \{-|s| / \alpha\}, \quad s \in \mathcal{S}$, denoted by $P_{S} \backsim L(0, \alpha)$. Note that for $P_{S} \backsim L(0, \alpha), \mathbb{E}_{P_{S}}|s|=\alpha$. We assume that the distortion measure is the magnitude-error distortion given by $d\left(s, s^{\prime}\right) \triangleq\left|s-s^{\prime}\right|$ for any $s, s^{\prime} \in \mathbb{R}$. The pdf for $k$-tuple source symbols is hence given by

$$
P_{S^{k}}(\mathbf{s})=\left[\frac{1}{2 \alpha}\right]^{k} \exp \left\{-\frac{\sum_{i=1}^{k}\left|s_{i}\right|}{\alpha}\right\}, \quad \mathbf{s} \in \mathcal{S}^{k}
$$

and the distortion for any $\mathbf{s} \triangleq\left(s_{1}, s_{2}, \ldots, s_{k}\right) \in \mathbb{R}^{k}, \mathbf{s}^{\prime} \triangleq$ $\left(s_{1}^{\prime}, s_{2}^{\prime}, \ldots, s_{k}^{\prime}\right) \in \mathbb{R}^{k}$ is given by $d^{(k)}\left(\mathbf{s}, \mathbf{s}^{\prime}\right) \triangleq \frac{1}{k} \sum_{i}\left|s_{i}-s_{i}^{\prime}\right|$.

For $P_{S} \backsim L(0, \alpha)$, the differential entropy and the ratedistortion function (under the magnitude-error distortion measure) are respectively given by $h\left(P_{S}\right)=1+\ln (2 \alpha)$ and $R\left(P_{S}, \Delta\right)=\max \left\{0, \ln \frac{\alpha}{\Delta}\right\}$. The Kullback-Leibler divergence between two MLS $\widehat{P}_{S} \backsim L(0, \widehat{\alpha})$ and $P_{S} \backsim L(0, \alpha)$ is equal to $D\left(\widehat{P}_{S} \| P_{S}\right)=\widehat{\alpha} / \alpha-\ln (\widehat{\alpha} / \alpha)-1$.

Lemma 1: Let $Q_{S}$ be an arbitrary pdf on $\mathcal{S}=\mathbb{R}$ such that $\underset{\mathbb{P}_{S}}{\mathbb{E}_{Q_{S}}}|s|=\alpha<\infty$. Consider two MLS $P_{S} \backsim L(0, \alpha)$ and $\widetilde{P}_{S} \backsim L(0, \widetilde{\alpha})$. Then

1) $h\left(Q_{S}\right) \leq h\left(P_{S}\right)$ with equality if and only if $Q_{S}=P_{S}$;

2) $D\left(Q_{S} \| \widetilde{P}_{S}\right) \geq D\left(P_{S} \| \widetilde{P}_{S}\right)$ with equality if and only if $Q_{S}=P_{S}$

3) $R\left(Q_{S}, \Delta\right) \leq R\left(P_{S}, \Delta\right)$ for any $\Delta>0$ with equality if $Q_{S}=P_{S}$ ('only if' holds when $\Delta \leq \alpha$ ).

\section{LAPLACIAN-TyPe Classes AND TyPe COVERING LEMMA}

For given $\alpha>0$ and $0<\epsilon<\alpha$, a Laplacian-type class $\mathcal{T}^{\epsilon}(\alpha)$ is defined as the set of all $k$-vectors $\mathbf{s} \in \mathbb{R}^{k}$ such that $\left|\sum_{i=1}^{k}\right| s_{i}|-k \alpha| \leq k \epsilon$, i.e.,

$$
\mathcal{T}^{\epsilon}(\alpha) \triangleq\left\{\mathbf{s}:\left|\sum_{i=1}^{k}\right| s_{i}|-k \alpha| \leq k \epsilon\right\} .
$$

The ( $k$-dimensional) conditional Laplacian-type class with respect to $\mathbf{s}^{*}=\left(s_{1}^{*} \cdots s_{k}^{*}\right)$ is defined by

$$
\mathcal{T}^{\epsilon}\left(\alpha \mid \mathbf{s}^{*}\right) \triangleq\left\{\mathbf{s}:\left|\sum_{i=1}^{k}\right| s_{i}-s_{i}^{*}|-k \alpha| \leq k \epsilon\right\} .
$$

Let $\operatorname{Vol}\{A\} \triangleq \int_{\mathbf{s} \in A} d \mathbf{s}$ be the volume of set $A$. As for the case of Gaussian-type classes, we can bound the volumes of the Laplacian-type class and the conditional Laplacian-type class as shown in the following lemma.

Lemma 2: For any $\alpha>\epsilon>0$ and any $\mathbf{s}^{*} \in \mathbb{R}^{k}$, we have

$$
\begin{aligned}
\operatorname{Vol}\left\{\mathcal{T}^{\epsilon}(\alpha)\right\} & \leq[2 e(\alpha+\varepsilon)]^{k}, \\
\operatorname{Vol}\left\{\mathcal{T}^{\epsilon}\left(\alpha \mid \mathbf{s}^{*}\right)\right\} & \geq\left[1-\frac{\alpha^{2}}{k \epsilon^{2}}\right]\left(2 \alpha e^{1-\frac{\epsilon}{\alpha}}\right)^{k} .
\end{aligned}
$$

It can also be shown that the probability of the type class $\mathcal{T}^{\epsilon}(\widetilde{\alpha})$, for $\widetilde{\alpha}>0$, under the Laplacian distribution $P_{S} \backsim$ $L(0, \alpha)$ is bounded by the exponential function

$$
P_{S}\left(\mathcal{T}^{\epsilon}(\widetilde{\alpha})\right) \leq \exp \left\{-k\left(\frac{\widetilde{\alpha}}{\alpha}-\ln \frac{\widetilde{\alpha}}{\alpha}-1+\zeta(\epsilon)\right)\right\}
$$

where $\zeta(\epsilon) \rightarrow 0$ as $\epsilon \rightarrow 0$. We next introduce the type covering lemma for Laplacian-type classes.

Lemma 3: (Type Covering Lemma) Given $\alpha>0$ and $\kappa>0$, for sufficiently small $\epsilon$ and for sufficiently large $k$, there exists a set $\mathcal{C} \subset \mathbb{R}^{k}$ of size $|\mathcal{C}| \leq \exp \left\{k\left[R\left(P_{S}, \Delta\right)+\zeta(\epsilon)\right]+\kappa\right\}$ such that every sequence in $\mathcal{T}^{\epsilon}(\alpha)$ is contained, for some $\mathbf{c} \in \mathcal{C}$, in the "cube" $B(\mathbf{c}, \Delta) \triangleq\left\{\mathbf{s}: \frac{1}{k} \sum_{i=1}^{k}\left|s_{i}-c_{i}\right| \leq \Delta\right\}$ of size $\Delta$ and centered at $\mathbf{c}$, where $R\left(P_{S}, \Delta\right)$ is the rate distortion function of Laplacian source $P_{S} \backsim L(0, \alpha)$ and $\zeta(\epsilon) \rightarrow 0$ as $\epsilon \rightarrow 0$.

The proof of this lemma, relying on a random-coding and geometric argument, is similar to the one proposed in [1], but differs in choosing the suitable conditional type class for the magnitude-error distortion measure.

Proof of Lemma 3: We start by assuming that $\alpha \geq \Delta$ since when $\alpha<\Delta$ the type $\mathcal{T}^{\epsilon}(\alpha)$ is covered by the cube $B(\mathbf{0}, \Delta)$ for $\epsilon$ sufficiently small $(\epsilon<\Delta-\alpha$ ), i.e., for $\alpha<\Delta$ and for $\epsilon<\Delta-\alpha$ there exists a code with size $|\mathcal{C}|=1$ that covers $\mathcal{T}^{\epsilon}(\alpha)$.

Construct a grid $X(\delta)$ of all vectors in the Euclidean space $\mathbb{R}^{k}$ whose components are integer multiples of $\delta$ for some small $0<\delta<\Delta$ (we set $\delta=\epsilon$ in the following) and consider the $k$-dimensional cubes of size $\delta$, centered at the grid points. Now we randomly (independently) choose $M$ vectors $\mathbf{c}^{(1)}, \ldots, \mathbf{c}^{(M)}$ in the set $\mathcal{T}^{\xi}(\alpha-(\Delta-\delta))$ according to the uniform pdf $P(\mathbf{c}) \triangleq 1 / \operatorname{Vol}\left\{\mathcal{T}^{\xi}(\alpha-(\Delta-\delta))\right\}$, where

$$
\begin{aligned}
& \xi \triangleq\left[1+\left(1-\frac{\Delta-\delta}{\alpha}\right)^{2}\right] \epsilon \text { and } \\
& \exp \left\{k\left[R\left(P_{S}, \Delta\right)+\zeta(\epsilon)\right]+\frac{\kappa}{2}\right\} \leq M \\
& \leq \exp \left\{k\left[R\left(P_{S}, \Delta\right)+\zeta(\epsilon)\right]+\kappa\right\} .
\end{aligned}
$$

Define the set $U(\Delta)$ by

$$
\begin{aligned}
& U(\Delta)=\left\{\mathbf{s} \in \mathcal{T}^{\epsilon}(\alpha) \bigcap X(\delta):\right. \\
& \left.\quad \frac{1}{k} \sum_{j=1}^{k}\left|s_{j}-c_{j}^{(i)}\right|>\Delta-\delta, \quad \text { for all } i=1,2, \ldots, M\right\} .
\end{aligned}
$$

Clearly, $U(\Delta)$ is a set of all grid points in set $\mathcal{T}^{\epsilon}(\alpha)$ which are not covered by the codewords in $\mathcal{C} \triangleq\left\{\mathbf{c}^{(1)}, \ldots, \mathbf{c}^{(M)}\right\}$ of size $M$ within distortion threshold $\Delta-\delta$. Now if we can show that $\mathbb{E}_{P}|U(\Delta)|<1$, where the expectation is taken under the uniform distribution $P(\mathbf{c})$, then there must exist a code for which $U(\Delta)$ is empty. For such code $\mathcal{C}, U(\Delta)$ is covered by the union of cubes $B\left(\mathbf{c}^{(i)}, \Delta-\delta\right), i=1,2, \ldots, M$, which then implies that, $\mathcal{T}^{\epsilon}(\alpha)$ is entirely covered by the union of cubes $B\left(\mathbf{c}^{(i)}, \Delta\right)$. According to the above random selection assumption,

$$
\begin{aligned}
= & \mathbb{E}_{P}\left\{\sum_{\mathbf{s} \in \mathcal{T}^{\epsilon}(\alpha) \cap X(\delta) \mid} \prod_{i=1}^{M} \Phi\left(\frac{1}{k} \sum_{j=1}^{k}\left|s_{j}-c_{j}^{(i)}\right|>\Delta-\delta\right)\right\} \\
= & \sum_{\mathbf{s} \in \mathcal{T}^{\epsilon}(\alpha) \cap X(\delta)} \prod_{i=1}^{M} \\
& {\left[1-P\left\{\mathbf{c}^{(i)}: \frac{1}{k} \sum_{j=1}^{k}\left|s_{j}-c_{j}^{(i)}\right| \leq \Delta-\delta\right\}\right] . }
\end{aligned}
$$


Now for each $\mathbf{s} \in \mathcal{T}^{\epsilon}(\alpha)$, we consider the conditional-type class $\mathcal{T}^{\epsilon}\left(D-\frac{D^{2}}{\alpha} \mid\left(1-\frac{D}{\alpha}\right)^{2} \mathbf{s}\right)$ where $D \triangleq \Delta-\delta<\alpha$. It can be readily verified that $\mathcal{T}^{\epsilon}\left(D-\frac{D^{2}}{\alpha} \mid\left(1-\frac{D}{\alpha}\right)^{2} \mathbf{s}\right) \subseteq$ $\mathcal{T}^{\xi}(\alpha-D)$ and

$$
\begin{aligned}
& \mathcal{T}^{\epsilon}\left(D-\frac{D^{2}}{\alpha} \mid\left(1-\frac{D}{\alpha}\right)^{2} \mathbf{s}\right) \\
\subseteq & \left\{\mathbf{c}^{(i)}: \frac{1}{k} \sum_{j=1}^{k}\left|s_{j}-c_{j}^{(i)}\right| \leq D\right\} .
\end{aligned}
$$

Since the codewords are distributed uniformly in $\mathcal{T}^{\xi}(\alpha-D)$, applying Lemma 2 and recalling that $\delta=\epsilon$ we have

$$
\begin{aligned}
& P\left\{\mathbf{c}^{(i)}: \frac{1}{k} \sum_{j=1}^{k}\left|s_{j}-c_{j}^{(i)}\right| \leq D\right\} \\
\geq & \frac{\operatorname{Vol}\left\{\mathcal{T}^{\epsilon}\left(D-\frac{D^{2}}{\alpha} \mid\left(1-\frac{D}{\alpha}\right)^{2} \mathbf{s}\right)\right\}}{\operatorname{Vol}\left\{\mathcal{T}^{\xi}(\alpha-D)\right\}} \\
\geq & \exp \left\{-k\left[\ln \frac{\alpha}{\Delta}+\zeta(\epsilon)\right]+o(k)\right\},
\end{aligned}
$$

where

$$
\zeta(\epsilon)=-\ln \frac{\Delta-\epsilon}{\Delta}+\ln \left(1+\frac{\epsilon}{\alpha-D}\right)+\frac{\xi \alpha}{D(\alpha-D)}
$$

and $o(k)=\ln \left[1-\frac{D-D^{2} / \alpha}{k \xi^{2}}\right]$. Substituting (5) into (4) yields

$$
\begin{aligned}
& \mathbb{E}_{P}|U(\Delta)| \\
\leq \quad & \left|\mathcal{T}^{\epsilon}(\alpha) \bigcap X(\delta)\right| \\
& {\left[1-\exp \left\{-k\left[\ln \frac{\alpha}{\Delta}+\zeta(\epsilon)\right]+o(k)\right\}\right]^{M} } \\
\leq \quad & {\left[\frac{2 e(\alpha+\epsilon)}{\delta}\right]^{k} } \\
& \exp \left\{-M \exp \left\{-k\left[\ln \frac{\alpha}{\Delta}+\zeta(\epsilon)\right]+o(k)\right\}\right\},
\end{aligned}
$$

where (6) holds since each codeword is independently selected and (7) follows from the inequality $(1-x)^{M} \leq e^{-M x}$ and the fact that the number of cubes in $\mathcal{T}^{\epsilon}(\alpha)$ is bounded by the ratio between the volumes of $\mathcal{T}^{\epsilon}(\alpha)$ and of a cube $\delta^{k}$. From (7) we note that for sufficiently small $\epsilon\left(\epsilon<D-D^{2} / \alpha\right), \delta=\epsilon$, and any given $\kappa>0$, there exists a set of codewords with size $M$ of exponential order $\exp \{k[\ln (\alpha / \Delta)+\zeta(\epsilon)]+\kappa\}$ (see (3)) such that $|U(\Delta)|=0$ as $k$ goes to infinity, which means that there exists a code of such exponential size covering $\mathcal{T}^{\epsilon}(\alpha)$ within distortion $\Delta$ for sufficiently large $k$.

\section{Application to the Lossy Source Coding of the MLS}

The lossy source coding theorem (e.g., [2], [6]) for a DMS with distribution $Q$ states that only $R(Q, \Delta)+\varepsilon$ bits per source symbol are needed to reproduce the source within the distortion threshold $\Delta$ with arbitrarily small probability of exceeding the distortion threshold $\Delta$, i.e., the probability of representing the source with a distortion larger than $\Delta$ asymptotically vanishes with the coding blocklength. The source coding excess distortion exponent describes the asymptotic behavior of the smallest possible probability of excess distortion as a function of the coding rate. In this section, we investigate the excess distortion exponent for the MLS.

A $(k, M)$ block source code for an MLS $P_{S}$ is a pair of mappings: $f_{k}: \mathbb{R}^{k} \longrightarrow\{1,2, \ldots, M\}$ and $\varphi_{k}$ : $\{1,2, \ldots, M\} \longrightarrow \mathbb{R}^{k}$. The probability of exceeding a given distortion threshold $\Delta>0$ for the code $\left(f_{k}, \varphi_{k}\right)$ is given by

$$
P_{\Delta}(k, M) \triangleq \int_{\mathbf{s}: d^{(k)}\left(\mathbf{s}, \varphi_{k}\left(f_{k}(\mathbf{s})\right)\right)>\Delta} P_{S^{k}}(\mathbf{s}) d \mathbf{s} .
$$

We call $P_{\Delta}(k, M)$ the probability of excess distortion. The code rate is defined by

$$
R(k, M) \triangleq \frac{1}{k} \ln M \quad \text { bits/source symbol. }
$$

Definition 1: For any $R>0$ and $\Delta>0$, the excess distortion exponent $F\left(R, P_{S}, \Delta\right)$ of the MLS $P_{S}$ is defined as the largest number $e$ for which there exists a sequence of $k$-length block codes $(k, M)$ with

$$
e \leq \liminf _{k \rightarrow \infty}-\frac{1}{k} \ln P_{\Delta}(k, M)
$$

and

$$
R \geq \limsup _{k \rightarrow \infty} \frac{1}{k} \ln M
$$

In the following we determine the excess distortion exponent $F\left(R, P_{S}, \Delta\right)$.

Theorem 1: For the MLS $P_{S} \sim L(0, \alpha)$ and distortion threshold $\Delta$, the excess distortion exponent satisfies

$$
F\left(R, P_{S}, \Delta\right) \geq \max \left\{0, \frac{e^{R} \Delta}{\alpha}-\ln \frac{e^{R} \Delta}{\alpha}-1\right\} .
$$

Remark: Clearly, this lower bound is nontrivial (positive) if $R>R\left(P_{S}, \Delta\right)$.

Sketch of Proof: For a given $\epsilon>0$ small enough, we partition the whole source space $\mathbb{R}^{k}$ by a sequence of Laplaciantype classes

$$
\mathcal{T}_{i} \triangleq \mathcal{T}^{\epsilon}(2 i \epsilon)=\left\{\mathbf{s}:\left|\sum_{i=1}^{k}\right| s_{i}|-2 k i \epsilon| \leq k \epsilon\right\},
$$

for $i=1,2, \cdots$, and the set $\mathcal{T}_{0} \triangleq\left\{\mathbf{s}: \sum_{i=1}^{k}\left|s_{i}\right| \leq k \epsilon\right\}$.

We employ a $\Delta$-admissible quantizer $\gamma_{k}^{(i)}: \mathcal{T}_{i} \rightarrow \mathcal{C}_{i}$ to encode each source message $\mathbf{s} \in \mathcal{T}_{i}$ into $\mathbf{c} \triangleq \gamma_{k}^{(i)}(\mathbf{s})$ such that $d^{(k)}(\mathbf{s}, \mathbf{c}) \leq \Delta$, where $\mathcal{C}_{i} \in \mathbb{R}^{k}$ is the codebook of $\gamma_{k}^{(i)}$ associated with $\mathcal{T}_{i}, i=0,1,2, \cdots$. For $\mathcal{T}_{0}$, there exists a $\Delta$-admissible quantizer with code size 1 , since trivially, we can encode all the sequences in $\mathcal{T}_{0}$ into $\mathbf{0}$. For $\mathcal{T}_{i}(i \geq 1)$, the type covering lemma (Lemma 3 ) ensures the existence of such a quantizer $\gamma_{k}^{(i)}$ for each $\mathcal{T}_{i}$ with code size $\left|\mathcal{C}_{i}\right| \leq$ $\exp \left\{k\left[R\left(P_{S}^{(i)}, \Delta\right)+\zeta(\epsilon)\right]+o(k)\right\}$, where $P_{S}^{(i)} \sim \mathcal{L}(0,2 i \epsilon)$, $\zeta(\epsilon)$ and $o(k)$ stand for some high order terms that $o(k) \rightarrow 0$ as $k \rightarrow \infty$ and $\zeta(\epsilon) \rightarrow 0$ as $\epsilon \rightarrow 0$. Next we employ a lossless 
source code $\left(\widehat{f}_{k}, \widehat{\varphi}_{k}\right): \widehat{f}_{k}: \bigcup_{i=0}^{\infty} \mathcal{C}_{i} \longrightarrow\left\{1,2, \ldots,\left\lceil e^{k R}\right\rceil\right\}$ and $\widehat{\varphi}_{k}:\left\{1,2, \ldots,\left\lceil e^{k R}\right\rceil\right\} \longrightarrow \bigcup_{i=0}^{\infty} \mathcal{C}_{i}$, and reproduce the source message $\widehat{\mathbf{c}}$.

Under the above coding scheme (the concatenation of the $\Delta$-admissible quantization and the lossless source coding), the probability of excess distortion is bounded by

$$
\begin{aligned}
P_{\Delta}(k, M) & =\sum_{i=0}^{\infty} \int_{\mathbf{s} \in \mathcal{T}_{i}: d^{(k)}(\mathbf{s}, \widehat{\mathbf{c}})>\Delta} P_{S^{k}}(\mathbf{s}) d \mathbf{s} \\
& \leq \sum_{i=0}^{\infty} \int_{\mathbf{s} \in \mathcal{T}_{i}: \widehat{\mathbf{c}} \neq \mathbf{c}} P_{S^{k}}(\mathbf{s}) d \mathbf{s} \\
& =\sum_{i=0}^{\infty} \sum_{\mathbf{c} \in \mathcal{C}_{i}: \widehat{\mathbf{c}} \neq \mathbf{c}} \underbrace{P_{S^{k}}\left(\mathcal{T}_{i}\right) P_{S^{k}}^{(i)}(\mathbf{c})}_{P(\mathbf{c})},
\end{aligned}
$$

where

$$
P_{S^{k}}^{(i)}(\mathbf{c}) \triangleq \frac{1}{P_{S^{k}}\left(\mathcal{T}_{i}\right)} \int_{\mathbf{s} \in \in \mathcal{T}_{i}: \gamma_{k}^{(i)}(\mathbf{s})=\mathbf{c}} P_{S^{k}}(\mathbf{s}) d \mathbf{s} .
$$

We note that (12) is exactly the probability of error for the lossless coding of the DMS with countable alphabet $\bigcup_{i=0}^{\infty} \mathcal{C}_{i}$ and distribution $P(\mathbf{c}) \triangleq P_{S^{k}}\left(\mathcal{T}_{i}\right) P_{S^{k}}^{(i)}(\mathbf{c})$ (the distribution of the output of the $\Delta$-admissible quantizer). Thus, there exists a sequence of lossless source codes $\left(\widehat{f}_{k}, \widehat{\varphi}_{k}\right)$ such that the probability of error is bounded by (see, e.g., [5, Ch. 5])

$$
P_{\Delta}(k, M) \leq \exp \{-k e(R, P)+o(k)\}
$$

for $k$ sufficiently large, where $e(R, P)$ is the lossless source coding error exponent for $P(\mathbf{c})$ and here is given by

$$
\begin{aligned}
& e(R, P) \\
= & \max _{\rho \geq 0}\left[\rho R-\frac{1+\rho}{k} \ln \sum_{i=0}^{\infty} P_{S^{k}}\left(\mathcal{T}_{i}\right)^{\frac{1}{1+\rho}} \sum_{\mathbf{c} \in \mathcal{C}_{i}} P_{S^{k}}^{(i)}(\mathbf{c})^{\frac{1}{1+\rho}}\right]
\end{aligned}
$$

and $o(k)$ stands for a generic term that vanishes as $k \rightarrow \infty$. By Jensen's inequality and the type covering lemma (Lemma 3 ), the sum over each $\mathcal{C}_{i}$ can be bounded by

$$
\begin{aligned}
& \sum_{\mathbf{c} \in \mathcal{C}_{i}} P_{S^{k}}^{(i)}(\mathbf{c})^{\frac{1}{1+\rho}} \leq\left|\mathcal{C}_{i}\right|^{\frac{\rho}{1+\rho}} \\
\leq & \exp \left\{\frac{\rho}{1+\rho}\left[k R\left(P_{S}^{(i)}, \Delta\right)+\zeta(\epsilon)\right]+o(k)\right\} .
\end{aligned}
$$

Note also that the probability of each $\mathcal{T}_{i}$ (including $\mathcal{T}_{0}$ ) is bounded by

$$
P_{S^{k}}\left(\mathcal{T}_{i}\right) \leq \exp \left\{-k\left[D\left(P_{S}^{(i)} \| P_{S}\right)+\zeta(\epsilon)\right]\right\} \quad i \geq 0 .
$$

Finally, using the fact [1] that

$$
\begin{aligned}
& \lim _{k \rightarrow \infty} \frac{1}{k} \ln \sum_{i=0}^{\infty} \exp [k(\ln (A i+B)-C i)+o(k)] \\
= & \sup _{i}[\ln (A i+B)-C i],
\end{aligned}
$$

where $A, B$, and $C$ are positive reals, and letting $\epsilon \rightarrow 0$, we can further lower bound $e(R, P)$ by

$$
e(R, P) \geq \frac{e^{R} \Delta}{\alpha}-\ln \frac{e^{R} \Delta}{\alpha}-1
$$

Therefore, we have demonstrated the existence of a sequence of concatenated lossy source codes $\left(\widehat{f}_{k} \circ \gamma_{k}^{(i)}, \widehat{\varphi}_{k}\right)$ such that

$$
P_{\Delta}(k, M) \leq \exp \left\{-k\left[\frac{e^{R} \Delta}{\alpha}-\ln \frac{e^{R} \Delta}{\alpha}-1\right]+o(k)\right\}
$$

for $k$ sufficiently large.

Corollary 1: The lower bound given in (11) is tight, i.e.,

$$
F\left(R, P_{S}, \Delta\right)=\frac{e^{R} \Delta}{\alpha}-\ln \frac{e^{R} \Delta}{\alpha}-1
$$

if $R>\ln \max \{\alpha / \Delta, 1\}$; Otherwise, $F\left(R, P_{S}, \Delta\right)=0$.

Proof: It is known from [4] that for the MLS source $P_{S} \sim$ $L(0, \alpha)$ and distortion threshold $\Delta$,

$$
F\left(R, P_{S}, \Delta\right)=\inf _{Q_{S}: R\left(Q_{S}, \Delta\right) \geq R} D\left(Q_{S} \| P_{S}\right),
$$

where the infimum is taken over all distributions $Q_{S}$ defined on $\mathbb{R}$. It suffices to show that (15) is indeed identical to our lower bound (11).

Consider the pdf $Q_{S}$ defined on $\mathbb{R}$ which is absolutely continuous with respect to $P_{S}$ and satisfies $R\left(Q_{S}, \Delta\right) \geq$ $R$. Suppose the expectation $\mathbb{E}|s|$ under $Q_{S}$ is equal to $\gamma$. According to Lemma 1, the Laplacian pdf $P_{S}^{*} \backsim L(0, \gamma)$ satisfies $D\left(P_{S}^{*} \| P_{S}\right) \leq D\left(Q_{S} \| P_{S}\right)$ and $R\left(P_{S}^{*}, \Delta\right) \geq$ $R\left(Q_{S}, \Delta\right) \geq R$. Therefore,

$$
\begin{aligned}
& \inf _{Q_{S}: R\left(Q_{S}, \Delta\right) \geq R} D\left(Q_{S} \| P_{S}\right) \\
& = \begin{cases}\frac{\gamma^{*}}{\alpha}-\ln \frac{\gamma^{*}}{\alpha}-1 & \text { for } R>R\left(P_{S}^{*} \| P_{S}\right) \\
0 & \text { otherwise, }\end{cases} \\
& P_{S}^{*} \backsim L(0, \gamma): R\left(P_{S}^{*}, \Delta\right) \geq R
\end{aligned}
$$

where $\gamma^{*}$ is determined by $R=\ln \left(\gamma^{*} / \Delta\right)$. This is exactly the exponent $F\left(R, P_{S}, \Delta\right)$ given by (14).

\section{REFERENCES}

[1] E. Arikan and N. Merhav, "Guessing subject to distortion," IEEE Trans. Inform. Theory, vol. 44, No. 3, pp. 1041-1056, May 1998.

[2] I. Csiszár and J. Körner, Information Theory: Coding Theorems for Discrete Memoryless Systems. New York: Academic, 1981.

[3] I. Csiszár, "The method of types," IEEE Trans. Inform. Theory, vol. 44, pp. 2505-2522, Nov. 1998.

[4] S. Ihara and M. Kubo, "Error exponent of coding for stationary memoryless sources with a fidelity criterion," IEICE Tran. Fundamentals, vol. E88-A, no. 5, pp 1339-1345, May. 2005.

[5] F. Jelinek, Probabilistic Information Theory, New York, McGraw Hill, 1968.

[6] K. Marton, "Error exponent for source coding with a fidelity criterion," IEEE Trans. Inform. Theory, vol. IT-20, pp. 197-199, Mar. 1974.

[7] R. C. Reininger and J. D. Gibson, "Distributions of the two-dimensional DCT coefficients for images," IEEE Trans. Commun., vol. 31, pp. 835839, June 1983.

[8] N. Tanabe and N. Farvardin, "Subband image coding using entropycoded quantization over noisy channels," IEEE J. Select. Areas Commun., vol. 10, pp. 926-943, June 1992.

[9] Y. Zhong, F. Alajaji, and L. L. Campbell, "On the excess distortion exponent for memoryless Gaussian source-channel pairs," Submitted to 2006 IEEE Int'l. Symp. Inform. Theory, Jan. 2006. 